\title{
Editorial
}

International Archives of
Allergy
Immunology

Published online: March 1, 2012

DOI: $\underline{10.1159 / 000335237}$

\section{A Silica-Induced Pulmonary Fibrosis Model: Are We Closer to ‘Real Life'?}

\section{E. Fireman}

Institute of Pulmonary and Allergic Diseases, National Service for Interstitial Lung Diseases, Tel Aviv Sourasky Medical Center, and Department of Epidemiology and Preventive Medicine, Sackler Faculty of Medicine, Tel Aviv University, Tel Aviv, Israel

Idiopathic pulmonary fibrosis (IPF) is a chronic and progressive lung disease that is associated with high mortality rates and is unresponsive to currently available treatments. The study of IPF in humans is complicated by the fact that its natural history is unknown and the evolution of the process is unclear. By the time patients seek medical treatment for symptoms the disease process is generally advanced [1]. A growing body of evidence suggests that, unlike other interstitial lung diseases, IPF is a distinct entity in which inflammation is a secondary and nonrelevant pathogenic partner. It has been suggested that IPF is characterized by a sequence of events that starts with alveolar epithelial microinjuries followed by the formation of fibroblastic foci and resulting in an exaggerated deposition of the extracellular matrix, which drives the destruction of the lung parenchyma architecture $[2,3]$. Animal models have been developed to study the evolution of fibrotic responses and these have identified a number of key cells, mediators and processes that are likely involved in human IPF. However, no current animal model is capable of recapitulating all of these cardinal manifestations of the human disease. As such, there are no appropriate experimental models of IPF, not only because we do not know its etiology, but also because all the traditional models start with an inflammatory reaction in spite of the fact that IPF is now accepted as being a noninflammatory disease.

\section{KARGER}

Fax +4161306 1234

E-Mail karger@karger.ch

www.karger.com
(C) 2012 S. Karger AG, Basel

$1018-2438 / 12 / 1583-0211 \$ 38.00 / 0$

Accessible online at:

www.karger.com/iaa
It is important to emphasize that the bleomycin model, the most ubiquitous experimental model of lung fibrosis, is a paradigm of the inflammatory route. Findings in animals sacrificed at early stages (i.e. 3 days postinstillation) are almost exclusively inflammatory, while those sacrificed at later stages (i.e. 14-21 days postinstillation) show both inflammation and fibrosis. Thus, the bleomycin model is useful for investigating the fibrotic response to acute lung injury and for evaluating the short-term treatment with various drugs, but not for mimicking human IPF [4].

In this issue of International Archives of Allergy and Immunology, Shimbori et al. [5] presented a very wellconducted study on silica-induced pulmonary fibrosis, and showed that treatment with a highly selective CysLT1 receptor antagonist (pranlukast) has antifibrotic effects that are independent of any effect on the acute inflammatory response. One of the most important findings of the present study is that long-term treatment (10 weeks) with pranlukast significantly attenuated the development of pulmonary fibrosis, while short-term treatment (2 weeks) failed to inhibit the initial increase in hydroxyproline content in the fibrotic lungs. These results demonstrate that the beneficial antifibrotic effect of the long-term pranlukast treatment regimen may be due to the inhibition of the progression of fibrosis rather than the inhibition of the onset of fibrosis. In addition, the number of inflam- 
matory cells in bronchoalveolar lavage fluids, the leukotriene content and the mRNA levels of cytokines were not affected by the short-term treatment regimen. It follows, therefore, that pranlukast has no therapeutic effect on the acute inflammatory response.

The use of silica aerosolization to induce pulmonary fibrosis has frequently been reported in the literature [6]. The instillation of mineral fibers into the rodent lung results in the development of fibrotic nodules that resemble lesions which develop in humans following some occupational exposures to mineral dust and particulate aerosols [7]. As with bleomycin-induced injury, the silica-induced fibrosis is strain dependent. The fibrotic response due to instillation of silica in mice is associated with limited and transient inflammation and overexpression of the antiinflammatory cytokine, IL-10; notably, anti-inflammatory therapies have no effect in this murine model [8]. Despite the fact that silica instillation induces a strong Th2 response in mice [9], these cytokines are not instrumental in the development of the disease [10]. In spite of the fact that the silica model is closer to the human condition, several limitations have prevented its wider use for studying IPF. For example, the silica-induced fibrosis lacks the characteristic IPF histology, with areas of fibrosis having no fibroblastic foci, temporal heterogeneity or hyperplastic epithelium. Furthermore, delivery of aero- solized silica is also problematic insofar as it requires expensive and specialized equipment. This model might actually be more suitable to reflect secondary fibrosis due to occupational exposure rather than idiopathic onset.

Another important novel finding of the present study is that the content of CysLTs in fibrotic lungs was markedly increased in silica-instilled mice, and that the 10 week pranlukast treatment attenuated both the progression of pulmonary fibrosis and the observed increases in CysLT content in fibrotic lungs. Although we believe that some drug targets for human disease can effectively be identified through animal studies (e.g. the transforming growth factor- $\beta$ pathway of ligand activation, receptor binding site and intracellular signal network), it has recently been pointed out that we know far less about the pathogenesis of human IPF than of experimental fibrosis in mice and rats $[11,12]$.

Shimbori et al. [5] have contributed a valuable heretofore unavailable opportunity to evaluate long-term induced pulmonary fibrosis in an animal model that comes closer to the human condition than earlier ones. Their having studied a molecule well recognized for human use in other pulmonary diseases may speed the achievement of the final goal of discovering effective treatment for idiopathic pulmonary processes in general and, especially, for occupational-induced fibrosis.

\section{References}

1 Kuhn C: Pathology; in Phan S, Thrall R (eds): Pulmonary Fibrosis. New York, Dekker, 1995, pp 59-83.

$>2$ Selman M, King TE, Pardo A: Idiopathic pulmonary fibrosis: prevailing and evolving hypotheses about its pathogenesis and implications for therapy. Ann Intern Med 2001; 134:136-151.

3 Katzenstein ALA, Fiorelli RF: Nonspecific interstitial pneumonia/fibrosis: histologic features and clinical significance. Am J Surg Pathol 1994;18:136-147.

$\checkmark 4$ Borzone G, Moreno R, Urrea R, Meneses M, Oyarzun M, Lisboa C: Bleomycin-induced chronic lung damage does not resemble human idiopathic pulmonary fibrosis. Am J Respir Crit Care Med 2001;163:1648-1653.
Shimbori C, Shiota N, Okunishi H: Pranlukast, a cysteinyl leukotriene type 11 receptor antagonist, attenuates the progression, but not the onset, of silica-induced pulmonary fibrosis in mice. Int Arch Allergy Immunol 2012;158:241-251.

6 Davis GS, Leslie KO, Hemenway DR: Silicosis in mice: effects of dose, time, and genetic strain. J Environ Pathol Toxicol Oncol 1998; 17:81-97.

7 Oberdorster G: Significance of particle parameters in the evaluation of exposure-doseresponse relationships of inhaled particles. Inhal Toxicol 1996;8:73-89.

8 Barbarin V, Nihoul A, Misson P, Arras M, Delos M, Leclercq I, Lison D, Huaux F: The role of pro- and anti-inflammatory responses in silica-induced lung fibrosis. Respir Res 2005;6:112.
Barbarin V, Xing Z, Delos M, Lison D, Huaux F: Pulmonary overexpression of IL-10 augments lung fibrosis and Th2 responses induced by silica particles. Am J Physiol Lung Cell Mol Physiol 2005;288:L841-L848.

10 Misson P, Brombacher F, Delos M, Lison D Huaux F: Type 2 immune response associated with silicosis is not instrumental in the development of the disease. Am J Physiol Lung Cell Mol Physiol 2007;292:L107-L113.

11 Chua F, Gauldie J, Laurent GJ: Pulmonary fibrosis: searching for model answers. Am J Respir Cell Mol Biol 2005;33:9-13.

12 Hunninghake GW, Schwarz MI: Does current knowledge explain the pathogenesis of idiopathic pulmonary fibrosis? A perspective. Proc Am Thorac Soc 2007;4:449-452. 